\title{
Fresh insight:polish the druggability of lipid metabolism for anti-renal fibrosis
}

\author{
yuanyuan Chen $^{1}$ and Sen Zhang ${ }^{1}$ \\ ${ }^{1}$ Chinese Academy of Medical Sciences and Peking Union Medical College
}

May 28, 2020

\begin{abstract}
Renal fibrosis can contribute to progressive damage both to renal structure and function. It is a common pathological process through which chronic kidney disease develops into kidney failure irrespective etiologies, and eventually leads to death. However, there is no available drug to treat renal fibrosis. Lipid aggregation and lipid toxicity within kidney always tightly accompanied chronic kidney disease as well as renal fibrosis. Moreover, accumulated studies have revealed that restoring the defective fatty acid oxidation in the kidney cells can reduce renal fibrosis. Thus, it is an important strategy to correct the dysfunctional lipid metabolism in the kidney by targeting critical regulators of lipid metabolism. This emerging direction brings ideas for the drug target determination to prevent or treat renal fibrosis, which may create bona fide drugs for this thorny disease burden. In this review, we highlight the potential "druggability" of lipid metabolism to resist renal fibrosis and provide current preclinical evidence, exemplified by some representative druggable targets and several other metabolic regulators with anti-renal fibrosis roles. Then we introduce the preliminary progress of noncoding RNAs and phytochemicals as promising anti-renal fibrosis drug targets or drugs from the perspective of lipid metabolism. Finally, we discuss the prospects and deficiencies of interfering with lipid reprogramming in the kidney.
\end{abstract}

Fresh insight:polish the druggability of lipid metabolism for anti-renal fibrosis Yuanyuan Chen ${ }^{1}$, Sen Zhang ${ }^{1, *}$

${ }^{1}$ Chinese Academy of Medical Sciences and Peking Union Medical College, Beijing, China.

* For corresponding author: Sen Zhang, Email:zhangs@imm.ac.cn, Phone: 8615201539761

Abstract

Renal fibrosis can contribute to progressive damage both to renal structure and function. It is a common pathological process through which chronic kidney disease develops into kidney failure irrespective etiologies, and eventually leads to death. However, there is no available drug to treat renal fibrosis. Lipid aggregation and lipid toxicity within kidney always tightly accompanied chronic kidney disease as well as renal fibrosis. Moreover, accumulated studies have revealed that restoring the defective fatty acid oxidation in the kidney cells can reduce renal fibrosis. Thus, it is an important strategy to correct the dysfunctional lipid metabolism in the kidney by targeting critical regulators of lipid metabolism. This emerging direction brings ideas for the drug target determination to prevent or treat renal fibrosis, which may create bona fide drugs for this thorny disease burden. In this review, we highlight the potential "druggability" of lipid metabolism to resist renal fibrosis and provide current preclinical evidence, exemplified by some representative druggable targets and several other metabolic regulators with anti-renal fibrosis roles. Then we introduce the preliminary progress of noncoding RNAs and phytochemicals as promising anti-renal fibrosis drug targets or drugs from the perspective 
of lipid metabolism. Finally, we discuss the prospects and deficiencies of interfering with lipid reprogramming in the kidney.

Key words: lipid metabolism, anti-renal fibrosis, targets, druggability

\section{Introduction}

Fibrosis is a pathological pathway that induces and aggravates multiple diseases, seriously devastating human health. The kidney is one of the mainly organs prone to be suffered from fibrosis, with a strikingly high incidence-second only to the liver (on average, the annual incidence of renal fibrosis exceeds $10 \%$ ) ${ }^{[1]}$. In the kidney, fibrosis often occurred in three parts: glomeruli, renal interstitium, and renal blood vessels (arteries and peripheral blood vessels) ${ }^{[2]}$. Tubular tubule is most susceptible to fibrosis and tubulointerstitial fibrosis may predict chronic kidney disease $(\mathrm{CKD})^{[3,4]}$. CKD resulted from different factors can develop fibrosis and progress to end-stage renal disease (ESRD). Therefore, blocking fibrosis, the fundamental pathway to ESRD will delay the progression of CKD. Although the mechanism of renal fibrosis is being gradually elucidated, in fact there are no available anti-renal fibrosis drugs. In recent years, growing evidence has emphasized the lipid accumulation and the following lipotoxicity in renal cells is a definite contributor to kidney injury. There is an intimate crosstalk between lipid metabolism and renal fibrosis, and it was demonstrated that lipid metabolism is a pivotal cause of fibrosis, not only in parallel to the fibrotic tendency. Moreover, restoring abnormal lipid metabolism within the kidney to a normal degree would effectively prevent and treat renal fibrosis ${ }^{[5-7]}$. A groundbreaking study deeply analyzed the relationship between transforming growth factor beta (TGF- $\beta$ ) signal and lipid metabolism in kidney fibrosis: TGF- $\beta 1$, one of the member of TGF- $\beta$ superfamily acting as a central profibrotic role in fibrosis, induced metabolic reprogramming characterized by downregulation of fatty acid oxidation (FAO), which resulted in lipid accumulation. While artificial restoration of FAO could significantly eliminate fibrosis ${ }^{[5]}$. This has unveiled the therapeutic potential of intervening lipid metabolism in renal fibrosis. Hence, targeting molecules involved in lipid metabolism pathway to resolve the metabolic dysfunction such as FAO deficiency can bring new methods for renal fibrosis treatment.

Herein, we introduce the crosstalk between lipid metabolism and renal fibrosis in recent years and especially discuss the emerging direct mediation of aberrant lipid metabolism in renal fibrosis. This summarized review on the "druggability" of lipid-related regulators and provide preclinical trial reference for further drug targets determination in this field, which is beneficial to guide the drug research and development for renal fibrosis.

\section{A brief overview of current mainstay mechanisms in renal fibrosis}

Renal fibrosis is an evolutionary and dynamic process with complex mechanisms and roughly considered to undertake four inseparable stages: priming, activation, execution and progression, which occur concomitantly occasionally and overlapped mutually ${ }^{[8]}$. Inflammation and epithelial damage are often the two major initial characteristics in renal fibrosis development. Generally, the kidney can trigger a protective inflammatory response when confronting stimulation or injury, but sustained chronic inflammation can incur inflammatory cells convergence to the kidney, including but not limited to macrophages, mast cells, $\mathrm{T}$ cells and extra fibroblast cells. They secrete fibrosis-related factors along with epithelial cells, podocytes, mesangial cells and endothelial cells, eventually breaking the balance between degradation and production of extracellular matrix (ECM), which generates irregularly gathering $\mathrm{ECM}^{[9]}$ (as seen in Fig. 1). For example, macrophages are recruited into the kidney by chemokines and activated by corresponding cytokines into M1 subtype or M2 subtype. M1, the classically activated proinflammatory phenotype, will produce a series of cytokines, growth factors (e.g. TGF- $\beta$, PDGF, FGF, IL-1 and TNF, etc.) and reactive oxygen species (ROS), to promote mesenchymal transformation of endothelial and epithelial cells, which results in the surge of myofibroblasts. Macrophages seem to also undergo macrophage-to-myofibroblast transition to function as ECM producers ${ }^{[10]}$. Cells secreting ECM are always termed myofibroblasts and their resource is extensive and incompletely clear yet, despite the proposed main myofibroblasts precursors (as seen in Fig. 1). Myofibroblasts can rapidly produce a large amount of ECM, hence fostering renal fibrosis and corresponding structural and functional damages ${ }^{[11]}$. It is currently believed that in renal fibrosis, the TGF- $\beta$-dominated pathway is the master regulatory mechanism ${ }^{[12-14]}$. Downstream of TGF- $\beta, \operatorname{smad} 2 / \operatorname{smad} 3$ are molecules that promote 
fibrosis and they form a complex with smad4 upon phosphorylation to upregulate the transcription of profibrogenic genes, while smad7 acts to be anti-fibrotic, so the broken balance between $\operatorname{smad} 2 / \operatorname{smad} 3 / \operatorname{smad} 4$ and smad7 will stimulate fibrogenesis ${ }^{[2]}$ (Fig. 2). In addition to the conventional TGF- $\beta /$ smad pathway, TGFBalso mediate fibrosis through signaling such as RhoA/ROCK, TRAF4/6, Ras/RAF, MAPK, JNK/ERK, PI3K/Akt, collectively referred to as non-canonical pathway. Other well-known signaling pathways WNT, Notch and Hedgehog have also been reported to coordinate in renal fibrosis and these pathways crosstalk with TGF- $\beta$ signals, increasing the complexity and obscurity of renal fibrosis mechanisms ${ }^{[15-18]}$.

According to clinical trials, anti-fibrotic druggable approaches in the TGF- $\beta$ pathway include antibodies, small molecules, siRNA, and oligonucleotides ${ }^{[19]}$. These drugs are mostly designed for fibrosis in lung, liver, skin fibrosis, and digestive system (https://clinicaltrials.gov/). To date, there is no drug specific for fibrosis within the kidney has obtained ideal outcome and some trials have already been withdrawn (eg, NCT00001959, NCT00878761 and NCT00464321). Wholly, the candidates in trials targeting TGF- $\beta$ have not progressed smoothly (NCT00878761). Connective tissue growth factor (CTGF) is a stromal cell protein induced by TGF- $\beta$ and is recognized as a profibrotic element. There are clinical trials of CTGF antibodies for the treatment of idiopathic pulmonary fibrosis (NCT00074698 and NCT01890265), but whether CTGF is necessary for the development of fibrosis in the kidney is not very clear yet ${ }^{[20-22]}$, and there is no clinical trial to evaluate the efficacy of CTGF antibody in anti-renal fibrosis. Therefore, finding alternative efficient targets other than TGF- $\beta$ signaling pathway is indeed an important and urgent strategy for preventing and treating renal fibrosis. Table 1 . has displayed the ongoing clinical trials about drugs for anti-renal fibrosis.

\section{Major lipid metabolism pathways involved in renal fibrosis}

The kidney is one of the organs with the strongest metabolic capacity. Unlike other organs or tissues, the kidney primarily uses lipid instead of glucose as the principle energy source and FAO is especially a critical metabolic step in the kidney. CD36 (cluster of differentiation 36), responsible for FA uptake, is expressed highly on the membrane of most cell types, including renal tubular epithelial cells ${ }^{[23]}$, podocytes ${ }^{[24]}$, mesangial cells $^{[25]}$ and macrophages ${ }^{[26]}$. Thus, CD36 is the most influential FA transporter in the kidney. The expression of CD36 is increased when kidney diseases proceed, which induces lipid overload and renal lipotoxicity ${ }^{[27]}$. In addition, fatty acid-binding protein (FABP) family and fatty acid transport proteins (FATP) family also participate in the cellular uptake of FA and other lipophilic substances. lipid entering the cell is deposited in the cytoplasm or taken up into the peroxisome or into the mitochondria (FAO rate-limiting step) by carnitine palmitoyl-transferase 1 (CPT1) and CPT2 (as seen in Fig. 3). Subsequently, $\beta$-oxidation of FA occurs and ATP is produced. Each molecule of FA can yield 106 ATP, while one molecule of carbohydrate can only offer 36 ATP maximally, so FAO is the chief energy supply for cell activities in some high-energyconsuming organs, especially the kidney ${ }^{[28]}$. Lipid uptake, synthesis and degradation are regulated by a series of transcription factors, involving peroxisome proliferator-activated receptors (PPARs), sterol regulatory element-binding transcription factor, SREBPs), farnesoid X receptor (FXR) and CCAAT enhancer binding protein (CCAAT/Enhancer-binding Protein $\alpha, \mathrm{C} / \mathrm{EBP} \alpha$ ), etc., and their gene expression has also been governed by some specific miRNAs whose expression changes during renal fibrosis ${ }^{[29-32]}$. Therefore, targeting enzyme, transporters, transcription factors, or miRNAs responsible for lipid metabolism to artificially regulate renal lipid metabolism can prevent or treat renal fibrosis.

\section{Treating renal fibrosis via interfering with lipid metabolism}

\subsection{Targeting representative regulators with preclinical evidence}

Based on the above putative ideas, the feasibility and effect of targeting renal lipid metabolism pathway to improve fibrosis were being explored in preclinical experiments. Here we discuss the necessary regulatory proteins, CD36, CPT1/2, SREBP1/2, PPARs, PGC-1 $\alpha$ and PCSK9 in lipid metabolism, which have been the representative mediators in kidney injury and fibrosis.

\subsubsection{CD36}

CD36, also known as scavenger receptor B2, is a transmembrane glycoprotein that performs as a receptor 
of long-chain fatty acids, oxidized lipids, advanced glycation end products and oxidized protein products ${ }^{[33]}$. As mentioned earlier, CD36 is the most important transporter that regulates fatty acid entry into cells, and it is overexpressed in different kidney pathological models to initiate the formation of lipid droplets and the development of fibrosis ${ }^{[34]}$. It was previously reported that gene intervention or drug treatment can reduce abnormally increased CD36 expression, ameliorate oxidative stress and inflammation, and inhibit TGF- $\beta$, which suggested CD36 a potential target for anti-renal fibrosis ${ }^{[35,36]}$. In recent years, considering the promising aspects of "targeting" lipid metabolism in fibrosis, researchers have further investigated possibility of CD36 as a drug target for anti-renal fibrosis. In the 5/6 nephrectomy with angiotensin II perfusion mouse model and unilateral ureteral obstruction (UUO) model, micropump containing CD3 antagonist 5A peptide can reduce inflammatory factors and chemokines, delay the progression of CKD and inhibit renal interstitial fibrosis ${ }^{[37]}$; Human CD36 overexpressed transgenic mice had obviously more proinflammatory components, profibrotic molecules and proteinuria than the wild mice during folic acid-induced (FAI) acute kidney injury; In diabetic mice model, CD36 also mediates the renal lipotoxicity and profibrotic effects of terminal protein oxidation products ${ }^{[27]}$. These results imply that CD36 regulates the development of fibrosis in various disease background in addition to CKD. In addition, soluble CD36 in serum is positively correlated with CD36 in the kidney and can be used as a biomarker to predict CKD. Therefore, in-depth study of the role of CD36 in renal physiology and pathology is of great value for the diagnosis and treatment of CKD and renal fibrosis. But there is a noteworthy problem that CD36 is highly expressed in many types of kidney cells, and their respective change in the expression level may cause different changes in phenotype. For example, in macrophages, CD36 is mainly related to the fibrosis signaling pathway, and interstitial fibrosis were significantly weakened in UUO mice after the specific knockout of macrophages CD36, while CD36 in distal renal tubular cells is involved in the binding of albumin and uptake, thus its abnormal expression mainly leads to the production of proteinuria ${ }^{[38]}$. Therefore, the specific targeting cell types should be considered when employing a CD36 inhibitor.

\subsubsection{CPT1/2}

CPT family proteins include CPT1 and CPT2, which are present on the outer and inner mitochondrial membranes, respectively. CPT1 have three subtypes, CPT1A, CPT1B and CPT1C. CPT1/2 is rate-limiting enzymes that transport fatty acids into the mitochondria and is indispensable for lipid catabolism. Downregulation or inactivation of CPT1 will block FAO, incurring fatty acids accumulate in the cytoplasm and cell necrosis. CPT1 deficiency will cause CKD and DKD, and renal tissue CPT1 expression is reduced in patients with renal fibrosis ${ }^{[5]}$.

The CPT1 activator C75, a synthetic tool compound, can inhibit fatty acid synthesis and improve renal function in a mouse model of FAI renal fibrosis. Thus CPT1 agonists are effective to reverse metabolic reprogramming for the blockade of fibrosis progression ${ }^{[5]}$. CPT1A is a prevailing enzyme converting acylCoA to acylcarnitine. It is the most widely distributed subtype among CPT1 members and has been intensely studied in cardiovascular diseases and malignant tumors ${ }^{[39]}$. In patients with CKD, the expression of CPT1A is negatively correlated with the degree of fibrosis. Animal experiments have shown that in a variety of renal fibrosis models, overexpression of CPT1 can reduce inflammation, TGF- $\beta$-induced epithelial cell damage and remarkably mitigate renal fibrosis. Gain of function of FAO mediated by upregulating CPT1 can correct the catastrophic lipid metabolic reprogramming, improve mitochondrial structure and metabolic activity in three common animal models of renal fibrosis, and profoundly prevent the process of renal fibrosis ${ }^{[40]}$. These prompt the opportunity of developing drugs for renal fibrosis as well as CKD based on CPT1. However, these studies on CPT2 in renal fibrosis are relatively fewer compared to CPT1. Recently, it has been found that the advanced glycation end products induced mitochondrial dysfunction through down-regulation of CPT2, impaired FAO, and caused renal fibrosis and DKD. Artificial overexpression of CPT2 could restore FAO and fibrosis-related genes to normal levels, manifesting that targeting CPT2 can help treat diabetic renal fibrosis ${ }^{[4]}$.

\subsubsection{SREBP1/2}

SREBPs (SREBP1a, SREBP1c and SREBP2) are a family of membrane-bound transcription factors bound 
to regulate lipid production. SREBP1a stimulates overall lipid synthesis, SREBP1c is responsible for fatty acid and triglyceride synthesis, and SREBP2 specifically controls sterol production ${ }^{[42]}$. Under normal conditions, SREBPs and their escort protein SCAP are locked by the complex consisting of lipid and the endoplasmic reticulum transmembrane protein ISIG, being anchored on the endoplasmic reticulum, which prevents SREBP from entering the nucleus to guide the transcription of genes involved in lipogenesis. Once the SCAP/SREBP complex detached from endoplasmic reticulum, it will enter Golgi apparatus freely, where SREBP was cleaved to release its transcriptionally active N-terminal fragments (Fig. 3). When SREBP is abnormally activated and ectopically located into nucleus, it will incur excessive lipid production and accumulation within the cells. Although the research on SREBPs is mainly concentrated on the field of cancer in which the elemental functions of SREBPs in regulating the proliferation and escape of tumor cells has been pointed out ${ }^{[43,44]}$,given the role of SREBPs in lipid homeostasis, it is not difficult to speculate that they will mediate the occurrence of renal fibrosis. In CKD and DKD patients, increased expression of SREBP1 and SREBP2 triggers kidney lipid deposition, lipotoxicity and fibrosis. Moreover, SREBP1 can regulate renal fibrosis through lipid independent pathway (directly acting on TGF- $\beta /$ Smad3 signal and inhibiting TGF- $\beta$ receptor degradation) $)^{[45]}$. By giving fatostatin, an inhibitor of SREBP in UUO mice, Maria Mustafa et al. demonstrated that blocking the signal transduction of SREBP1/2 on lipid production and TGF- $\beta 1$ can obviously reduce inflammation, necrosis and fibrosis in the kidney of UUO mice ${ }^{[46]}$. While recently in another group, Richard Van Krieken et al. declared that fatostatin did not alleviate proteinuria and renal fibrosis in diabetic mice, and it also aggravated secretion of inflammatory factors and pro-inflammatory factors in normal mice ${ }^{[47]}$. This discrepancy may attribute to the different background of disease model. Therefore, the role of SREBPs in renal pathology and the renal protective function of fatostatin need to be evaluated in more nephrotic models.

\subsubsection{PPARs}

PPARs belong to the type II nuclear hormone receptor superfamily and are divided into three subtypes, PPAR- $\alpha, \beta(\delta)$ and $\gamma$. They can serve as transcription factors binding to the response element within the promoter of genes related to glucose and lipid metabolism ${ }^{[48]}$. PPARs agonists such as fibrates are often used in the treatment of metabolic diseases ${ }^{[49]}$. The activation of PPAR- $\alpha$ and PPAR- $\beta$ enhances the decomposition of triglycerides and FAO, so the PPAR- $\alpha$ agonists and PPAR- $\alpha / \beta$ dual agonists are mainly applied to reduce blood lipids ${ }^{[50]} ; \mathrm{PPAR}-\gamma$ controls adipogenesis and adipocyte differentiation, insulin sensitivity and glucose metabolism. Its agonists have become prevalent drugs for type 2 diabetes ${ }^{[51,52]}$.

Increasing experiments have manifested that intervention of PPARs can regulate the occurrence and development of renal fibrosis. In the animal model of tubulointerstitial fibrosis, the expression of PPAR- $\alpha$ was significantly reduced, and the PPAR- $\alpha$ agonist BAY PP1, but not fenofibrate, could remarkably attenuate fibrosis through restoring PPAR- $\alpha$ level in renal tubular cells without adverse effects ${ }^{[53]}$. During the aging proceeding of the body, the degree of renal fibrosis gradually increases and renal function declines. Ki Wung Chung et al found that impaired PPAR- $\alpha$ pathway accelerates aging kidney fibrogenesis in mice ${ }^{[6]}$. This group later reported that giving MHY2013, a PPAR $\alpha / \beta$ dual agonist to aging rats can conduce to elimination of lipid aggregation and renal fibrosis in rats kidney ${ }^{[54]}$, These results confirmed that PPARs agonists have a delaying effect on renal dysfunction. Regarding renal fibrosis, recent studies have further shown that PPAR- $\alpha$ agonist fibrates possess visible therapeutic effects. In the context of renal transplantation-induced fibrosis, the administration of fenofibrate in the rat model of kidney transplantation can suppress EMT induced by oxidative stress and relieve renal fibrosis through directly improving FA metabolism ${ }^{[55]}$.

In parallel with this, PPAR- $\gamma$ participates in the maintenance of renal metabolism homeostasis ${ }^{[32]}$ and PPAR- $\gamma$ agonists have also been reported to have similar effects in a few literatures. Recently, Hye-Soon Kim and others found that lobelgitazone, a drug mainly used for diabetes, can inhibit the TGF- $\beta / \mathrm{Smad} 3$ pathway to discourage UUO-induced renal fibrosis, displaying kidney protective roles ${ }^{[56,57]}$; Another two PPAR- $\gamma$ agonists, rosiglitazone can inhibit EMT and tubulointerstitial fibrosis via antagonizing ROS in $\mathrm{DKD}^{[56]}$; pioglitazone can reduce TGF- $\beta 1$-induced renal fibrosis in mice by inhibiting EGR-1, STAT3 and AP- ${ }^{[58]}$, indicating that PPAR- $\gamma$ may be a practical target for the treatment of chronic kidney disease and 
fibrosis in the future. Besides, Increasing the indication of PPAR- $\gamma$ agonist, a new method of old medicine, can shorten the research and development cycle and reduce the cost of scientific research. However, in the UUO renal fibrosis model, Kawai T and Zhang Y et al.'S respective studies on the therapeutic effect of pioglitazone reached the opposite conclusion. Therefore, whether PPAR- $\gamma$ will anti-renal fibrosis requires further study ${ }^{[59,60]}$.

\section{$3.1 .5 \Pi \Gamma^{\prime \prime}-1 \alpha$}

Peroxisome proliferator-activated receptor- $\gamma$ coactivator (PGC)- $1 \alpha$ comes from PGC- 1 family, and the other two members of PGC- 1 are PGC-1 $\beta$ and PGC-1-related coactivator. PGC- $1 \alpha$ is a co-activator of PPARs and bound to stimulating mitochondrial and peroxisomal biogenesis. Plus that PPARs can affect the regulation of lipid synthesis and transport genes by interacting with PPARs, thus PGC- $1 \alpha$ is also an important cell lipid balancer and energy regulator ${ }^{[61]}$. PGC- $1 \alpha$ expression is often reduced in aging or diseased fibrotic kidneys, corresponding to a weakened cell metabolism ${ }^{[62]}$. Like PPARs, it is not difficult to surmise that PGC- $1 \alpha$ also affects renal fibrosis ${ }^{[63]}$. Administration of PPAR- $\gamma$ agonist rosiglitazone to DKD mice with fibrosis to increase the expression of PGC- $1 \alpha$ can reduce the production of ROS and protect kidney from injury ${ }^{[63]}$. Seung Hyeok Han and others reported that PGC-1 $\alpha$ was the target gene of Notch. After being directly targeted by Notch, PGC- $1 \alpha$ transcription was blocked, and then kidney cells encountered FAO defects. Restoring PGC- $1 \alpha$ gene expression can destroy Notch mediated kidney damage and fibrosis ${ }^{[64]}$. However, Jue-Long Wang et al silenced PGC- $1 \alpha$ by siRNA, which broke the positive feedback loop between TGF- $\beta$ and PGC-1 $\alpha$, and significantly decreased the expression of fibronectin and collagen $\mathrm{I}^{[65]}$. This was concluded that inhibition of PGC- $1 \alpha$ in renal tubular cells contributes to anti-fibrosis, contrary to the previous results. It is the first time to declare that PGC- $1 \alpha$ promotes fibrosis by acting on TGF $\beta$-RI, PI3K/Akt, and p38 MAPK. These studies have implied that intervention in PGC- $1 \alpha$ can change renal fibrosis, but the opposite results also obscure the role of PGC- $1 \alpha$ in the kidney.

\subsubsection{PCSK9}

PCSK9 is a gene closely related to hypercholesterolemia, and its encoded PCSK9 protein mainly exists in the liver. In the kidney, PCSK9 is also expressed at a lower level. PCSK9 binds to the low-density lipoprotein receptor (LDL-R) on the cell surface to form a complex, which will undergo cellular internalization for lysosomal degradation, resulting in reduced LDC-R. Since LDC-R is necessary to clear low-density lipoprotein cholesterol (LDL-C), deficiency in LDC-R will induce LDL-C overload. Accordingly, PCSK9 is a key molecule in regulating blood LDL-C. The inhibitor of PCSK9 (PCSK9i) is principally used in lipid-lowering therapy to regulate blood lipid metabolism and improve cardiovascular function ${ }^{[66]}$. Alirocumab and evolocumab are currently available PCSK9, both of which are monoclonal antibodies that bind to PCSK9. Several studies on CKD have shown that PCSK9i has renal protective effects. The application of PCSK9i in kidney disease mainly focuses on lowering the cholesterol level of CKD patients and reducing the risk of CKD complications such as cardiovascular disease ${ }^{[67,68]}$. There are few studies on PCSK9i employment in renal fibrosis. Through literature search we found only one study showed that the PCSK9Q $\beta-003$ vaccine could enhance fatty acid oxidation via its own original lipid regulation effect, ameliorating hypercholesterolemia and renal fibrosis in UUO and N-nitro-L-arginine methyl ester mice model ${ }^{[69]}$. In more detail, PCSK9Q $\beta$ 003 vaccine profoundly upregulated CD35, CPT1A, PPAR $\alpha / \gamma$, PGC- $1 \alpha$ and SREBP2, while downregulated TGF- $\beta /$ smad3 in kidney, representing a likely anti-fibrosis mechanism of FAO pathway. This study provides an elegant incentive that if PCSK9i, which has been approved for sale, can indeed hinder the development of renal fibrosis, this drug repositioning manner of PCSK9i will circumvent the target confirmation stage and provide new drugs to this field faster and more economically. Of course, whether PCSK9i can treat renal fibrosis as a new indication needs further preclinical trials and clinical trials to determine. Additionally, whether the anti-fibrosis PSK9 antibody mainly acts on the kidney cells themselves or the PCSK9 highly expressed liver remains to be corroborated, for that PCSK9 expression in the kidney is only mild.

\subsection{Other regulators with emerging role in treating renal fibrosis}

In addition to the typical lipid metabolism regulatory molecules in renal fibrosis mentioned above, recently 
the role of other glycolipid metabolism regulating proteins in renal fibrosis has also been preliminarily discovered, and they may continue be excavated as drug targets.

FABP 4 is one of the subtypes of FABP, which is responsible for the fatty acids transport and can downregulate its target gene PPAR- $\gamma^{[70]}$. In vitro use of FABP4 siRNA or inhibitors and in vivo FSBP4 knockdown can enhance PPAR- $\gamma$ signaling, improve lipid metabolism and inflammation, and reduce renal interstitial fibrosis through PPAR- $\gamma /$ ACOX1/CPT1 and PPAR- $\gamma / \mathrm{NF}-\varkappa \mathrm{B} / \mathrm{ICAM}$ pathways, respectively ${ }^{[71]}$. Numerous studies have proved that complement is a causative factor for a variety of CKD and acute kidney disease, and complement components including $\mathrm{C} 3$ and $\mathrm{C} 5$ are also involved in renal fibrosis ${ }^{[72-74]}$. In DKD, C5a inhibition can downregulate DGAT1 and SREBP-1. C5a inhibitor NOX-D21 reduced tubular sclerosis and improved lipid metabolism ${ }^{[75]}$, which suggested that antibody drug therapy for complement may also prevent renal fibrosis. ATF6 $\alpha$ is a transcription factor reported to participate in the lipid metabolism regulation in recent years. ATF $6 \alpha$ may downregulate PPAR- $\alpha$, mediate lipid aggregation and mitochondrial dysfunction, and promote fibrotic factors such as CTGF, a-SMA and collagen I secretion or expression ${ }^{[76]}$. The specific mechanism was examined in a model of unilateral ischemia-reperfusion injury-induced fibrosis that ATGF $\alpha$ in proximal renal tubular cells was activated and then entered the nucleus, acting as an inhibitor of PPAR- $\alpha$ to reduce the expression of PPAR- $\alpha$, which in turn led to down-regulation of CPT2, the target gene of PPAR- $\alpha$. For that CPT2 is an elemental transporter that mediates the entry of fatty acids into mitochondria, ATG $\alpha$ can influence FAO and mitochondrial functional homeostasis, inducing lipid aggregation/lipotoxicity, and following cell necrosis and CTGF expression, finally leading to fibrosis ${ }^{[76]}$. The subsequent use of fenofibrate to reverse ATF6 $\alpha$-mediated lipotoxicity and fibrosis in mouse kidneys further verified the mechanism of ATF $6 \alpha /$ PPAR- $\alpha /$ CPT2. Certainly, it was not clarified how ATGF $\alpha$ was activated. In addition, as the author of this study said, the role of ATF6 $\alpha$ in different disease models had two opposite situations: kidney protection and kidney damage aggravation, despite that in chronic and long-term pathological conditions such as CKD, ATF6 $\alpha$ generally tends to be non-adaptive activated state to disturb lipid metabolism. Liver kinase B1 (LKB1) is a serine kinase that regulates adipogenesis and differentiation. Seung Hyeok Han and others reported that the expression of phosphorylated LKB1 was decreased in the renal tissue of patients with renal fibrosis, which indicated that there was a link between LKB1 and renal fibrosis. Then they found that LKB1 deficiency would induce impaired renal glucose and fatty acid utilization, mediated by AMPK and PPAR- $\alpha^{[77]}$. Whether LKB1 can control fibrosis needs further verification. Autophagy is also tightly related to lipid metabolism. Therefore, autophagy may affect fibrosis by regulating lipid metabolism. In the process of renal fibrosis, autophagy activation often coexists with lipid aggregation. Preliminary studies have found that the autophagy protein beclin-1 can congregate in the endoplasmic reticulum, initiate the generation of autophagosomes, and promote the cellular deposition of lipid droplets, which may be resulted from the accumulation of free fatty acids, so the lipotoxicity caused by autophagy in the kidney will motivate fibrotic phenotype ${ }^{[78]}$ and targeting axis "autophagy-metabolism-fibrosis" may offer an alternative method. HIF- $1 \alpha$ is a key factor regulating cell lipid metabolism and lipid accumulation, thus provoking fibrosis by promoting ECM accumulation ${ }^{[79-81]}$. The mitochondrial uncoupling protein (UCP2) is also involved in the regulation of mitochondrial functional activity and lipids metabolism especially FAO. Qing Ke et al. demonstrated that UCP2 and HIF- $1 \alpha$ are involved in the disorder of lipid metabolism related to renal fibrosis in an acute ischemia-reperfusion induced renal fibrosis model. In renal tubular cells, UCP2 can stabilize HIF- $1 \alpha$ by regulating mitochondrial respiration and oxygen content, thereby regulating lipid accumulation and ECM, controlling the occurrence and development of fibrosis, which may be related to PPAR- $\alpha$ and CPT1A. Other molecules such as FATP2 ${ }^{[82]}$,TFAM ${ }^{[83]}$ and VEGFB ${ }^{[84]}$ have also been shown to influence the occurrence and development of nephropathy and fibrosis by regulating lipid metabolism. For example, FATP2 mediates the uptake of albumin-bound non-esterified fatty acids by distal renal tubular cells, avoiding the lipotoxicity of non-esterified fatty acids. Although deeply studies on these emerging regulators are still lacking, they may represent a novel series of components in the axis of "lipid metabolism-renal fibrosis".

\subsection{Lipid metabolism related non-coding RNAs}

Targeting non-coding RNAs, upstream of regulatory factors in lipid metabolism pathway, to treat renal fibrosis have acquired encouraging achievement preclinically. 
miRNAs are a class of non-coding RNAs that regulate gene expression transcriptionally via binding to mRNAs, and play a key role in cellular physiology and disease pathology, including metabolic programs ${ }^{[85,86]}$ and $\mathrm{CKD}^{[87,88]}$, respectively. Although there are relatively few studies on miRNAs in renal fibrosis, it can be speculated from the existing research results that miRNAs may be novel targets for anti-fibrosis. Moreover, miRNAs engage in kidney metabolism via monitoring specific genes, hence mediation of fibrogenesis in metabolic manner. In this part, we solely focus on the axis of miRNAs-lipid metabolism-renal fibrosis to conduct the topic of this review. MiR-21 is the first miRNA that has been shown to link lipid metabolism with renal fibrosis. MiR-21 targets PPAR- $\alpha$, Mpv17l (a gene that inhibits the production of ROS) and reck (inhibits metalloproteinases) and then reduces their expression, thus disrupting the balance of lipid metabolism, oxidative stress and cell proliferation and accelerating renal damage and renal fibrosis, while systematic knockout of miR-21 precludes the pathological role of miR-21 in UUO and unilateral ischemia-reperfusion mice model ${ }^{[89]}$. Practically, antisense oligonucleotides (ASOs) targeting miRNA have been initially utilized in preclinical studies to evaluate the therapeutic potential of miRNAs for renal fibrosis. Ivan G. Gomez et al. found that miR-21 can increase intracellular lipid aggregation and ROS production by silencing PPAR $\alpha$ and inhibiting antioxidant proteins. Subsequently, they further developed highly specific ASOs targeting miR-21, which selectively positioned in the kidney of mice model and knocked down miR-21 after administered subcutaneously. MiR-21 ASO significantly improved the proteinuria of Alport nephropathy (a hereditary glomerular disease, its clinical manifestation is chronic glomerulonephritis) model, and inhibited glomerular sclerosis and renal interstitial fibrosis. These therapeutic effects are related to the enhanced mitochondrial functional activity, reinforced mitochondrial FAO activity and reduced mitochondrial ROS production ${ }^{[90]}$. Note that nowadays the anti-miR21 oligonucleotide RG-012 has entered the clinical trial phase I (NCT03373786) and is tested in patients with Alport syndrome by subcutaneous injection. Although it is not specifically for renal fibrosis, RG-012 may also be developed into a drug for the treatment of renal fibrosis considering that miR21 genuinely dictate renal fibrosis. On the other hand, miR-21 from serum or urine can reflect renal fibrosis, and there are clinical trials to assess its reliability as a biomarker (NCT03780101). MiR-9-5p, a miRNA that functions to maintain homeostasis of organ functions, can prevent fibrosis of organs including the kidney. Compared with the mice injected only lentivirus vector, the renal interstitial fibrosis in UUO mouse model injected lentivirus carrying miR-9-5p was profoundly alleviated, with upregulation of genes mainly related to FAO, TCA cycle, glycolysis, OXPHOX, and mitochondrial function that are down-regulated during fibrosis. Among which, miR-9-5p prevented the decline of CPT1A level, and it was further found that PGC-1 $\alpha$ was necessary for miR-9-5p to exert renal protective effect. These illuminated the metabolic reprogramming effect of miR-9-5p in renal fibrosis, especially the regulation of cellular lipid metabolism pathway ${ }^{[91]}$. Similarly, miR-33, a miRNA known to be encoded by the region within the SREBP gene and highly expressed in the kidney, can promote fibrosis and inflammation in DKD through the NF- $x$ B/TGF- $\beta$ pathway ${ }^{[92]}$. Based on this, Nathan L. Price et al. used pH low insertion peptide to carry miR-33 peptide nucleic acid inhibitors. This tool enabled miR-33 targeted delivery to the kidney, an acidic environment. Then miR-33 target genes, CPT1A and CROT (an enzyme catalyzing the reversible transfer of fatty acyl groups) gained increased expression, and cellular lipid aggregation eliminated in kidney, which effectively dampened renal fibrosis. This study reveals the opportunity of targeting miRNA in kidney to treat fibrosis ${ }^{[93]}$.

Long non-coding RNAs (lncRNAs) are another type of non-coding RNAs with a length greater than $200 \mathrm{dt}$. They are able to manicipate the development of various diseases via regulating gene expression transcriptionally, post-transcriptionally and epigenetically. A few lncRNAs has been characterized in renal fibrosis, such as Erbb4-IR ${ }^{[13]} 、 \operatorname{lnc}-$ TSI $^{[94]} 、$ CYB4P1-PS1-001 ${ }^{[95]}$, lncRNA ZEB1-AS1 ${ }^{[96]}$ and lncRNA TUG1, etc,Among them, TUG1 could upregulate PGC-1 $\alpha$ expression through interacting with the upstream region of the PGC$1 \alpha$ gene to promote the binding of PGC1- $\alpha$ to its promoter, which partially reversed the high glucose-induced mitochondrial destruction in podocyte and facilitated energy metabolism ${ }^{[97]}$. The direct effect on PGC- $1 \alpha$ endued TUG1 with the ability to regulate mitochondrial biosynthesis and energy metabolism activity, suggesting that engineered TUG1 are likely to treat renal fibrosis.

Due to the controversy of PPARs agonists as discussed before, direct targeting miRNAs or lncRNAs in kidney 
may be a better selection. At present, siRNA and ASOs drugs targeting mRNA have been on the market for the treatment of nervous system diseases; some drugs targeting specific miRNA and lncRNA are also undergoing clinical trials. Additionally, in terms of drug delivery, liposomes and other nanoparticles modified with specific peptide chains to achieve highly specific targeting of miRNAs and lncRNAs will help reduce undesirable effects within other organs and tissues ${ }^{[98]}$. Therefore, designing kidney-specific anti-fibrotic drugs from non-coding RNA may be a promising area in the future. However, adequate experiments are demanded to clarify the regulatory network of a candidate miRNA or lncRNA and their targeting security remains to be seen.

\section{Phytochemicals}

Natural plant-derived bioactive molecules, also called phytochemicals, are a rich source of medicines with a long history, which have been exploited as an adjunct therapy for numberless diseases, including CKD. A large sample retrospective cohort survey showed that Chinese medicines, Rheum officinale Baill, Salvia miltiorrhiza Bunge and Bunge can indeed improve life quality and prolong survival of patients with $\mathrm{CKD}^{[99]}$. Dioscin is a biomolecule from common edible plant in daily life, dioscoreae rhizome. It has been reported for many times that dioscin has the effect of resisting nephrotoxicity and preventing renal injury ${ }^{[100-102]}$. Likewise, our group have discovered anti-fibrosis and anti-oxidation effect of coumarin glycosides from hydrangea paniculate in diabetic nephropathy and will delve into the details of its mechanism in addition to the confirmed TGF$\beta /$ smad pathway. Recently, dioscin was found to regulate TGF- $\beta 1 /$ Smad pathway by upregulating SIRT3, and impede fructose-induced renal fibrosis, involving inflammatory, lipid metabolism and oxidative stress signals alteration ${ }^{[103]}$; Shen Shuai II Recipe (SSR) is a common clinical prescription for the treatment of CKD. It can facilitate renal blood circulation and oxygen consumption by upregulating NF- $x \mathrm{~B} / \mathrm{TNF}-\alpha$ pathway. Likewise, SSR can activate the SIRT1 acetylation pathway to reduce acetylated smad3 and inhibit the activation of NLRP3, which curbs profibrotic and proinflammatory trends ${ }^{[104]}$. A review of traditional Chinese medicine and renal fibrosis can be found elsewhere ${ }^{[105]}$.

Herein, we offer an overview and opinion of phytochemicals in renal fibrosis especially from the lipid metabolic perspective. AO is a natural plant traditionally used for diuresis and anti-edema. The formula of Chinese medicine containing $\mathrm{AO}$ is considered to have anti-atherosclerosis, anti-diabetes and anti-nephropathy effects. $\mathrm{AO}$ also has the properties of improving lipid metabolism and anti-fibrosis, but the exact involved molecules are not yet clear. Recently, Fang Dou et al. initially explored the mechanism of AO's anti-renal fibrosis using lipidomics and found that AO can improve the lipid metabolism disorder and renal fibrosis in CKD by partially restoring the metabolism of FA and triglycerides in CKD rats. This elaborated the potential of natural plants to treat renal fibrosis from the viewpoint of glycolipid metabolism, and provided a metabolic way to find natural plants against renal fibrosis. Due to the crucial role of lipid metabolism and the widely application of traditional Chinese medicine in renal fibrosis model, it is necessary to study whether the natural plant molecules with metabolic regulation ability can resist renal fibrosis. For example, quercetin has long been reported to inhibit renal oxidative stress ${ }^{[106]}$, via regulating specific miRNAs ${ }^{[107]}$ or Hedgehog signaling ${ }^{[108]}$ to reduce EMT and fibrosis. Meanwhile, quercetin has therapeutic effects in metabolic diseases, especially in diabetes as well as its complications DKD, through the SCAP-SREBP2-LDLr pathway to improve lipid metabolism (lowering serum cholesterol, triglycerides and low density lipoprotein cholesterol), which relieves lipid droplet overburden and lowers blood urea nitrogen and blood glucose concentration; this suggests that quercetin may realize its anti-renal fibrosis effect through improving lipid metabolism ${ }^{[109]}$. Resveratrol is a polyphenol compound widely believed to have anti-aging, anti-diabetic ${ }^{[110]}$, vascular protection ${ }^{[111]}$ and neuroprotective ${ }^{[112]}$ functions. Earlier studies have shown that resveratrol can activate SIRT1 to deacetylate PGC- $1 \alpha$, thus enhance PGC- $1 \alpha$ gene expression and protein levels ${ }^{[13]}$, and reduce fibrotic marker protein level through AMPK/SIRT1/PGC- $1 \alpha$ pathway ${ }^{[14]}$;Resveratrol also regulates PPAR- $\alpha$ and PPAR- $\gamma$ to control cholesterol uptake and renal lipotoxicity ${ }^{[115,116]}$, and preclinical experiments prove that this phytochemical can be used as an anti-renal fibrosis drug within a certain dose range ${ }^{[117]}$. Mechanically, these facts reflect that PPARs and PGC- $1 \alpha$ may be the pivotal targets for resveratrol in anti-fibrosis. Metformin, derived from the natural plant lilac, serve as a preferred drug for type 2 diabetes. Furthermore, metformin influences multiple metabolic diseases progress and reinforces resistance to fibrosis in various organs, these 
two functions possess subtle connection. For example, metformin converts cellular metabolic phenotype of lung fibroblasts and upregulates the gene expression of PPAR- $\gamma$ and BMP2, and stimulates PPAR- $\gamma$ phosphorylation and its activities, which accelerates the removal of intracellular lipid droplets and tissue fibrosis by reversing cellular lipid metabolism reprogramming ${ }^{[18]}$. In the kidney, metformin can significantly reduce FAI fibrosis through the TGF- $\beta 1$ pathway, but whether it also reprogram metabolism in kidney to the similar extent and which metabolic pathways are mainly modified to exert its function requires more explanation ${ }^{[119]}$.

From the typical cases we have discussed above, it may be a followable direction to explore and select antirenal fibrosis drugs from Chinese medicines treasure that possess ability to regulate lipid metabolism. In turn, we can discover the mechanism of action for phytochemicals in renal fibrosis from metabolic angle, which will supplement insufficient mechanical research of phytochemicals. Therefore, phytochemicals deserve more attention.

\section{Glucose metabolism related reprogramming}

During the literature investigation, we noticed that there are a few studies on glucose metabolism and renal fibrosis. Albeit in the incipient stage, we still embrace them to reflect the current research trends to introduce anti-fibrosis target involved in glucose metabolism.

Although the energy supply manner of renal cells, especially renal tubular epithelial cells, is mainly lipid metabolism, glucose metabolism still belongs to one of the integral metabolic approach in the kidney. Changes in both glucose metabolism and fibrosis always arise concomitantly, indicating that these two procedures may have mutual mediation. Metabolic reprogramming is one of the ways in which cells have been transformed. Under non-hypoxic conditions, cancer cells employ aerobic glycolysis to boost glucose absorption, but reduce mitochondrial glucose oxidation, hence Warburg effect, which provides special or mutated metabolic program to support rapid cell growth. In renal fibrosis, glucose metabolism also undergoes similar reprogramming in addition to mentioned abnormal lipid metabolism. Renal glycolysis-related genes and protein levels are elevated when suffering fibrogenesis, and there is a positive correlation between the degree of pyruvate kinase M2 (PKM2, glycolytic rate-limiting enzyme) positive myofibroblasts and the severity of renal fibrosis. Aerobic glycolysis can provoke myofibroblast activation, while inhibiting aerobic glycolysis can prevent TGF$\beta$-induced myofibroblast activation in vitro, and inhibit UUO-induced renal fibrosis in vivo. PKM2 siRNA or glycolysis inhibitor shikonin and 2-deoxyglucose can reduce UUO-induced renal fibrosis via destroying PKM2 mediated aerobic glycolysis ${ }^{[120]}$. Similarly, it had recently been substantiated that expression of PKM2 and another critical glycolytic enzyme, human glandular kallikrein 2, would increase in renal fibrocytes and animal models; increased glycolysis of podocytes promoted cell proliferation and dedifferentiation, while inhibition of glycolysis restored podocyte structure and function and slowed down the development of fibrosis ${ }^{[121]}$. Corresponding glycolysis inhibitors could control renal interstitial fibrosis by regulating the differentiation of fibroblasts and tubular cells ${ }^{[22]}$, which further confirmed the role of glycolysis in fibrogenesis. Therefore, using targeted inhibitors to correct Warburg effect, metabolic switch from oxidative phosphorylation to aerobic glycolysis, in the kidney may also help to resist fibrotic proceeding.

\section{Summary and Outlook}

Aberrant intra-renal lipid metabolism is indeed a universal characteristic and pathological factor of renal fibrosis, therefore, finding drugs that can be inserted into renal lipid metabolism can interfere with renal fibrosis. In this article, we review the advancement of representative lipid metabolism regulators in recent years, discussing the anti-fibrotic effects of existing drugs or inhibitors against these candidate targets in preclinical animal models. The evaluations acquired from the preliminary studies have shown that the "druggable" lipid metabolism has potential to inhibit renal fibrosis. Among other lipid metabolism-related molecules, gene therapy based on miRNAs and phytochemicals have also been introduced, because there are gradually studies supporting that they connect kidney metabolism and fibrosis and have the possibility to be anti-fibrotic drugs. At the same time, although glucose metabolism is not the leading metabolic mode in the kidney, there are a few reports that glycolysis is also a seemingly contributor or mediator in renal 
fibrogenesis, so we have mentioned their budding roles in this area.

In the field of oncology, metabolic research is undoubtedly a hot spot, scientists are confident to develop new antineoplastic drugs from the perspective of metabolic reprogramming. In the study of fibrosis, it is also likely to find new drugs targeting metabolic dysregulation, and to achieve drug repositioning based on existing compound with targets in metabolism. The mechanism of fibrosis is particularly complex, far from clear conclusion, but now preclinical experiments have shown promising aspects of lipid metabolism such as FAO molecular pathway in treating renal fibrosis model. These should be timely valued by more researchers in the near future, because anti-renal fibrosis drugs targeting the traditional TGF- $\beta$ pathway result to be ineffective and nonspecific, so we must make a shift now. On the other hand, despite that lipid metabolism affects the physiology of the kidney to a great extent, research on finding a target in anti-renal fibrosis from this perspective are still lacking, and there has not been a determined suitable drug target yet. Therefore, it is urgent and essential to explore a set of weighting networks for the regulation of renal lipid metabolism and find the most critical targetable molecules in renal fibrosis. In addition, the considerable research is ongoing that modifying metabolic reprogramming to overcome other diseases such as fibrosis in other organs and cancer. It will provide an opportune reference for the prevention or treatment of fibrosis in the kidney by targeting metabolic pathway molecules; Besides, pharmaceutical preparations and delivery technology are also crucial to help to target specifically kidney tissues and even the exact cell types.

\section{Funding}

This word was supported by the Drug Innovation Major Project of China [grant number 2018ZX09711001002-010]; Chinese Academy of Medical Sciences Innovation Fund for Medical Sciences [grant number 2016I2M-3-011]; and Beijing Natural Science Foundation [grant numbers 7202138, 7181007].

\section{Competing interests}

None.

\section{Reference}

1 Zhao X, Kwan JYY, Yip K, Liu PP, Liu F-F. Targeting metabolic dysregulation for fibrosis therapy. Nature Reviews Drug Discovery 2019: 1-19.

2 Humphreys BD. Mechanisms of renal fibrosis. Annual review of physiology 2018; 80: 309-26.

3 Wilson PC, Kashgarian M, Moeckel G. Interstitial inflammation and interstitial fibrosis and tubular atrophy predict renal survival in lupus nephritis. Clinical Kidney Journal 2017; 11: 207-18.

4 Hewitson TD, Holt SG, Smith ER. Progression of Tubulointerstitial Fibrosis and the Chronic Kidney Disease Phenotype - Role of Risk Factors and Epigenetics. Frontiers in Pharmacology 2017; 8.

5 Kang HM, Ahn SH, Choi P, Ko Y-A, Han SH, Chinga F, et al . Defective fatty acid oxidation in renal tubular epithelial cells has a key role in kidney fibrosis development. Nature medicine 2015; 21: 37.

6 Chung KW, Lee EK, Lee MK, Oh GT, Yu BP, Chung HY. Impairment of PPARalpha and the Fatty Acid Oxidation Pathway Aggravates Renal Fibrosis during Aging. J Am Soc Nephrol 2018; 29: 1223-37.

7 Dorotea D, Koya D, Ha H. Recent Insights Into SREBP as a Direct Mediator of Kidney Fibrosis via Lipid-Independent Pathways. Frontiers in Pharmacology 2020; 11: 265.

8 Liu Y. Cellular and molecular mechanisms of renal fibrosis. Nat Rev Nephrol 2011; 7: 684-96.

9 Djudjaj S, Boor P. Cellular and molecular mechanisms of kidney fibrosis. Mol Aspects Med 2019; 65: $16-36$.

10 Wang Y-Y, Jiang H, Pan J, Huang X-R, Wang Y-C, Huang H-F, et al . Macrophage-to-myofibroblast transition contributes to interstitial fibrosis in chronic renal allograft injury. Journal of the American Society of Nephrology 2017; 28: 2053-67. 
11 Meng XM. Inflammatory Mediators and Renal Fibrosis. Adv Exp Med Biol 2019; 1165: 381-406.

12 Chen L, Yang T, Lu D-W, Zhao H, Feng Y-L, Chen H, et al . Central role of dysregulation of TGF$\beta /$ Smad in CKD progression and potential targets of its treatment. Biomedicine \& Pharmacotherapy 2018; 101: $670-81$.

13 Feng M, Tang PM-K, Huang X-R, Sun S-F, You Y-K, Xiao J, et al . TGF- $\beta$ mediates renal fibrosis via the Smad3-Erbb4-IR long noncoding RNA axis. Molecular Therapy 2018; 26: 148-61.

14 Li H, Cai H, Deng J, Tu X, Sun Y, Huang Z, et al . TGF- $\beta$-mediated upregulation of Sox9 in fibroblast promotes renal fibrosis. Biochimica Et Biophysica Acta (BBA)-Molecular Basis of Disease 2018; 1864: $520-32$.

15 Edeling M, Ragi G, Huang S, Pavenstädt H, Susztak K. Developmental signalling pathways in renal fibrosis: the roles of Notch, Wnt and Hedgehog. Nature Reviews Nephrology 2016; 12: 426.

16 He W, Dai C, Li Y, Zeng G, Monga SP, Liu Y. Wnt/beta-catenin signaling promotes renal interstitial fibrosis. Journal of the American Society of Nephrology : JASN 2009; 20: 765-76.

17 Bielesz B, Sirin Y, Si H, Niranjan T, Gruenwald A, Ahn S, et al . Epithelial Notch signaling regulates interstitial fibrosis development in the kidneys of mice and humans. The Journal of clinical investigation 2010; 120: 4040-54.

18 Fabian SL, Penchev RR, St-Jacques B, Rao AN, Sipilä P, West KA, et al . Hedgehog-Gli pathway activation during kidney fibrosis. The American journal of pathology 2012; 180: 1441-53.

19 Rauchman M, Griggs D. Emerging strategies to disrupt the central TGF- $\beta$ axis in kidney fibrosis. Translational Research 2019.

20 Montford JR, Furgeson SB. A new CTGF target in renal fibrosis. Kidney international 2017; 92: 784-6.

21 Nagai Y, Matoba K, Kawanami D, Takeda Y, Akamine T, Ishizawa S, et al . ROCK2 regulates TGF- $\beta$ induced expression of CTGF and profibrotic genes via NF- $\varkappa \mathrm{B}$ and cytoskeleton dynamics in mesangial cells. American Journal of Physiology-Renal Physiology 2019; 317: F839-F51.

22 Miguel V, Tituana J, Herrero JI, Herrero L, Serra D, Cuevas P, et al . Renal tubule Cpt1a overexpression mitigates kidney fibrosis by restoring mitochondrial homeostasis. bioRxiv 2020.

23 Hou Y, Wu M, Wei J, Ren Y, Du C, Wu H, et al . CD36 is involved in high glucose-induced epithelial to mesenchymal transition in renal tubular epithelial cells. Biochemical and biophysical research communications 2015; 468: 281-6.

24 Hua W, Huang H-z, Tan L-t, Wan J-m, Gui H-b, Zhao L, et al . CD36 mediated fatty acid-induced podocyte apoptosis via oxidative stress. PloS one 2015; 10.

25 Hughes J, Liu Y, Van Damme J, Savill J. Human glomerular mesangial cell phagocytosis of apoptotic neutrophils: mediation by a novel CD36-independent vitronectin receptor/thrombospondin recognition mechanism that is uncoupled from chemokine secretion. The Journal of Immunology 1997; 158: 4389-97.

26 Pennathur S, Pasichnyk K, Bahrami NM, Zeng L, Febbraio M, Yamaguchi I, et al . The macrophage phagocytic receptor CD36 promotes fibrogenic pathways on removal of apoptotic cells during chronic kidney injury. The American journal of pathology 2015; 185: 2232-45.

$27 \mathrm{Li} \mathrm{X}$, Zhang T, Geng J, Wu Z, Xu L, Liu J, et al . Advanced oxidation protein products promote lipotoxicity and tubulointerstitial fibrosis via CD36/ $\beta$-catenin pathway in diabetic nephropathy. Antioxidants \& redox signaling 2019; 31: 521-38.

28 Meyer C, Nadkarni V, Stumvoll M, Gerich J. Human kidney free fatty acid and glucose uptake: Evidence for a renal glucose-fatty acid cycle. American Journal of Physiology-Endocrinology And Metabolism 1997; 273: E650. 
29 Wang M. Kidney miR-33 controls fatty acid oxidation. Nature Reviews Nephrology 2020; 16: 66-.

30 Hou X, Tian J, Geng J, Li X, Tang X, Zhang J, et al . MicroRNA-27a promotes renal tubulointerstitial fibrosis via suppressing PPAR $\curlyvee$ pathway in diabetic nephropathy. Oncotarget 2016; 7: 47760.

31 Wang XX, Jiang T, Shen Y, Adorini L, Pruzanski M, Gonzalez FJ, et al . The farnesoid X receptor modulates renal lipid metabolism and diet-induced renal inflammation, fibrosis, and proteinuria. American journal of physiology. Renal physiology 2009; 297: F1587-F96.

32 Lyu Z, Mao Z, Li Q, Xia Y, Liu Y, He Q, et al . PPAR $\curlyvee$ maintains the metabolic heterogeneity and homeostasis of renal tubules. EBioMedicine 2018; 38: 178-90.

33 Yang X, Okamura DM, Lu X, Chen Y, Moorhead J, Varghese Z, et al . CD36 in chronic kidney disease: novel insights and therapeutic opportunities. Nature Reviews Nephrology 2017; 13: 769.

34 Susztak K, Ciccone E, McCue P, Sharma K, Böttinger EP. Multiple metabolic hits converge on CD36 as novel mediator of tubular epithelial apoptosis in diabetic nephropathy. PLoS medicine 2005; 2.

35 Yang YL, Lin SH, Chuang LY, Guh JY, Liao TN, Lee TC, et al . CD36 is a novel and potential antifibrogenic target in albumin-induced renal proximal tubule fibrosis. Journal of cellular biochemistry 2007; 101: 735-44.

36 Okamura DM, Pennathur S, Pasichnyk K, Lopez-Guisa JM, Collins S, Febbraio M, et al . CD36 regulates oxidative stress and inflammation in hypercholesterolemic CKD. Journal of the American Society of Nephrology 2009; 20: 495-505.

37 Souza ACP, Bocharov AV, Baranova IN, Vishnyakova TG, Huang YG, Wilkins KJ, et al . Antagonism of scavenger receptor CD36 by $5 \mathrm{~A}$ peptide prevents chronic kidney disease progression in mice independent of blood pressure regulation. Kidney international 2016; 89: 809-22.

38 Baines RJ, Chana RS, Hall M, Febbraio M, Kennedy D, Brunskill NJ. CD36 mediates proximal tubular binding and uptake of albumin and is upregulated in proteinuric nephropathies. American Journal of Physiology-Renal Physiology 2012; 303: F1006-F14.

39 Schlaepfer IR, Joshi M. CPT1A-mediated Fat Oxidation, Mechanisms, and Therapeutic Potential. Endocrinology 2020; 161.

40 Miguel V, Tituana J, Herrero JI, Herrero L, Serra D, Cuevas P, et al . Renal tubule Cpt1a overexpression mitigates kidney fibrosis by restoring mitochondrial homeostasis. bioRxiv 2020: 2020.02.18.952440.

41 Lee J, Hyon J-Y, Min JY, Huh YH, Kim HJ, Lee H, et al . Mitochondrial carnitine palmitoyltransferase 2 is involved in Ne-(carboxymethyl)-lysine-mediated diabetic nephropathy. Pharmacological Research 2020; 152: 104600 .

42 Horton JD, Goldstein JL, Brown MS. SREBPs: activators of the complete program of cholesterol and fatty acid synthesis in the liver. The Journal of clinical investigation 2002; 109: 1125-31.

43 Wen YA, Xiong X, Zaytseva YY, Napier DL, Vallee E, Li AT, et al . Downregulation of SREBP inhibits tumor growth and initiation by altering cellular metabolism in colon cancer. Cell Death Dis 2018; 9: 265.

44 Chen J, Wu Z, Ding W, Xiao C, Zhang Y, Gao S, et al . SREBP1 siRNA enhance the docetaxel effect based on a bone-cancer dual-targeting biomimetic nanosystem against bone metastatic castration-resistant prostate cancer. Theranostics 2020; 10: 1619.

45 Dorotea D, Koya D, Ha H. Recent Insights Into SREBP as a Direct Mediator of Kidney Fibrosis via Lipid-Independent Pathways. Frontiers in pharmacology 2020; 11: 265-.

46 Mustafa M, Wang TN, Chen X, Gao B, Krepinsky JC. SREBP inhibition ameliorates renal injury after unilateral ureteral obstruction. American Journal of Physiology-Renal Physiology 2016; 311: F614-F25. 
47 Van Krieken R, Marway M, Parthasarathy P, Mehta N, Ingram AJ, Gao B, et al . Inhibition of SREBP With Fatostatin Does Not Attenuate Early Diabetic Nephropathy in Male Mice. Endocrinology 2018; 159: 1479-95.

48 Tyagi S, Gupta P, Saini AS, Kaushal C, Sharma S. The peroxisome proliferator-activated receptor: A family of nuclear receptors role in various diseases. J Adv Pharm Technol Res 2011; 2: 236-40.

49 Han L, Shen W-J, Bittner S, Kraemer FB, Azhar S. PPARs: regulators of metabolism and as therapeutic targets in cardiovascular disease. Part I: PPAR- $\alpha$. Future cardiology 2017; 13: 259-78.

50 Lakhia R, Yheskel M, Flaten A, Quittner-Strom EB, Holland WL, Patel V. PPAR $\alpha$ agonist fenofibrate enhances fatty acid $\beta$-oxidation and attenuates polycystic kidney and liver disease in mice. American Journal of Physiology-Renal Physiology 2018; 314: F122-F31.

51 Bermúdez V, Finol F, Parra N, Parra M, Pérez A, Peñaranda L, et al . PPAR-gamma agonists and their role in type 2 diabetes mellitus management. American journal of therapeutics 2010; 17: 274-83.

52 Choi S-S, Kim E-S, Jung J-E, Marciano DP, Jo A, Koo JY, et al . PPAR $\curlyvee$ antagonist Gleevec improves insulin sensitivity and promotes the browning of white adipose tissue. Diabetes 2016; 65: 829-39.

53 Boor P, Celec P, Martin IV, Villa L, Hodosy J, Klenovicsova K, et al . The peroxisome proliferatoractivated receptor-alpha agonist, BAY PP1, attenuates renal fibrosis in rats. Kidney Int 2011; 80: 1182-97.

54 Chung KW, Ha S, Kim SM, Kim DH, An HJ, Lee EK, et al . PPARalpha/beta Activation Alleviates Age-Associated Renal Fibrosis in Sprague Dawley Rats. J Gerontol A Biol Sci Med Sci 2020; 75: 452-8.

55 Wang Y, Pang L, Zhang Y, Lin J, Zhou H. Fenofibrate improved interstitial fibrosis of renal allograft through inhibited epithelial-mesenchymal transition induced by oxidative stress. Oxidative medicine and cellular longevity 2019; 2019.

56 Bae KH, Seo JB, Jung YA, Seo HY, Kang SH, Jeon HJ, et al . Lobeglitazone, a Novel Peroxisome Proliferator-Activated Receptor $\gamma$ Agonist, Attenuates Renal Fibrosis Caused by Unilateral Ureteral Obstruction in Mice. Endocrinol Metab (Seoul) 2017; 32: 115-23.

57 Kim H-S, Bae KH, Jung GS, Ham HJ, Park BY, Choi YK, et al . 19th European Congress of Endocrinology. (BioScientifica).

58 Németh Á, Mózes MM, Calvier L, Hansmann G, Kökény G. The PPAR $\curlyvee$ agonist pioglitazone prevents TGF- $\beta$ induced renal fibrosis by repressing EGR-1 and STAT3. BMC nephrology 2019; 20: 245.

59 Kawai T, Masaki T, Doi S, Arakawa T, Yokoyama Y, Doi T, et al . PPAR- $\gamma$ agonist attenuates renal interstitial fibrosis and inflammation through reduction of TGF- $\beta$. Laboratory investigation 2009; 89: 47-58.

60 Zhang Y, Wang J, Zhou Q-d, Zhang C-h, Li Q, Huang S, et al . Peroxisome proliferator-activated receptor- $\gamma$ agonist pioglitazone fails to attenuate renal fibrosis caused by unilateral ureteral obstruction in mice. Journal of Huazhong University of Science and Technology [Medical Sciences] 2016; 36: 41-7.

61 Liang H, Ward WF. PGC-1alpha: a key regulator of energy metabolism. Advances in physiology education 2006; 30: 145-51.

62 Lee G, Uddin MJ, Kim Y, Ko M, Yu I, Ha H. PGC-1alpha, a potential therapeutic target against kidney aging. Aging Cell 2019; 18: e12994.

63 Zhang L, Liu J, Zhou F, Wang W, Chen N. PGC-1alpha ameliorates kidney fibrosis in mice with diabetic kidney disease through an antioxidative mechanism. Mol Med Rep 2018; 17: 4490-8.

64 Han SH, Wu M-y, Nam BY, Park JT, Yoo T-H, Kang S-W, et al . PGC-1 $\alpha$ protects from notch-induced kidney fibrosis development. Journal of the American Society of Nephrology 2017; 28: 3312-22. 
65 Wang JL, Chen CW, Tsai MR, Liu SF, Hung TJ, Yu Ju H, et al . Antifibrotic role of PGC-1alpha-siRNA against TGF-beta1-induced renal interstitial fibrosis. Exp Cell Res 2018; 370: 160-7.

66 Kaufman TM, Warden BA, Minnier J, Miles JR, Duell PB, Purnell JQ, et al . Application of PCSK9 Inhibitors in Practice. Circ Res 2019; 124: 32-7.

67 Charytan DM, Sabatine MS, Pedersen TR, Im K, Park JG, Pineda AL, et al. Efficacy and Safety of Evolocumab in Chronic Kidney Disease in the FOURIER Trial. Journal of the American College of Cardiology 2019; 73: 2961-70.

68 González Sanchidrián S, Labrador Gómez PJ, Aguilar Aguilar JC, Davin Carrero E, Gallego Domínguez S, Gómez-Martino Arroyo JR. Evolocumab for the treatment of heterozygous familial hypercholesterolemia in end-stage chronic kidney disease and dialysis. Nefrologia : publicacion oficial de la Sociedad Espanola Nefrologia 2019; 39: 218-20.

69 Wu D, Zhou Y, Pan Y, Li C, Wang Y, Chen F, et al . Vaccine Against PCSK9 Improved Renal Fibrosis by Regulating Fatty Acid beta-Oxidation. J Am Heart Assoc 2020; 9: e014358.

70 Hotamisligil GS, Bernlohr DA. Metabolic functions of FABPs - mechanisms and therapeutic implications. Nature Reviews Endocrinology 2015; 11: 592.

71 Qiao Y, Liu L, Yin L, Xu L, Tang Z, Qi Y, et al . FABP4 contributes to renal interstitial fibrosis via mediating inflammation and lipid metabolism. Cell death \& disease 2019; 10: 1-12.

72 Cui J, Wu X, Song Y, Chen Y, Wan J. Complement C3 exacerbates renal interstitial fibrosis by facilitating the M1 macrophage phenotype in a mouse model of unilateral ureteral obstruction. American Journal of Physiology-Renal Physiology 2019; 317: F1171-F82.

73 Liu Y, Wang K, Liang X, Li Y, Zhang Y, Zhang C, et al . Complement C3 Produced by Macrophages Promotes Renal Fibrosis via IL-17A Secretion. Front Immunol 2018; 9: 2385-.

74 Rangan GK, Pippin JW, Couser WG. C5b-9 regulates peritubular myofibroblast accumulation in experimental focal segmental glomerulosclerosis. Kidney international 2004; 66: 1838-48.

75 Yiu WH, Li RX, Wong DWL, Wu HJ, Chan KW, Chan LYY, et al . Complement C5a inhibition moderates lipid metabolism and reduces tubulointerstitial fibrosis in diabetic nephropathy. Nephrology Dialysis Transplantation 2017; 33: 1323-32.

76 Jao TM, Nangaku M, Wu CH, Sugahara M, Saito H, Maekawa H, et al . ATF6alpha downregulation of PPARalpha promotes lipotoxicity-induced tubulointerstitial fibrosis. Kidney Int 2019; 95: 577-89.

77 Han SH, Malaga-Dieguez L, Chinga F, Kang HM, Tao J, Reidy K, et al . Deletion of Lkb1 in renal tubular epithelial cells leads to CKD by altering metabolism. Journal of the American Society of Nephrology 2016; 27: 439-53.

78 Yan Q, Song Y, Zhang L, Chen Z, Yang C, Liu S, et al . Autophagy activation contributes to lipid accumulation in tubular epithelial cells during kidney fibrosis. Cell death discovery 2018; 4: 39.

79 Higgins DF, Kimura K, Bernhardt WM, Shrimanker N, Akai Y, Hohenstein B, et al . Hypoxia promotes fibrogenesis in vivo via HIF-1 stimulation of epithelial-to-mesenchymal transition. The Journal of clinical investigation 2007; 117: 3810-20.

80 Kimura K, Iwano M, Higgins DF, Yamaguchi Y, Nakatani K, Harada K, et al . Stable expression of HIF$1 \alpha$ in tubular epithelial cells promotes interstitial fibrosis. American Journal of Physiology-Renal Physiology 2008; 295: F1023-F9.

81 Bensaad K, Favaro E, Lewis CA, Peck B, Lord S, Collins JM, et al . Fatty acid uptake and lipid storage induced by HIF-1 $\alpha$ contribute to cell growth and survival after hypoxia-reoxygenation. Cell reports 2014; 9: $349-65$. 
82 Khan S, Cabral PD, Schilling WP, Schmidt ZW, Uddin AN, Gingras A, et al . Kidney proximal tubule lipoapoptosis is regulated by fatty acid transporter-2 (FATP2). Journal of the American Society of Nephrology 2018; 29: 81-91.

83 Huang S, Park J, Qiu C, Chung KW, Li S-y, Sirin Y, et al . Jagged1/Notch2 controls kidney fibrosis via Tfam-mediated metabolic reprogramming. PLoS biology 2018; 16: e2005233.

84 Falkevall A, Mehlem A, Palombo I, Sahlgren BH, Ebarasi L, He L, et al . Reducing VEGF-B signaling ameliorates renal lipotoxicity and protects against diabetic kidney disease. Cell Metabolism 2017; 25: 713-26.

$85 \mathrm{Yu} \mathrm{Y,} \mathrm{Du} \mathrm{H,} \mathrm{Wei} \mathrm{S,} \mathrm{Feng} \mathrm{L,} \mathrm{Li} \mathrm{J,} \mathrm{Yao} \mathrm{F,} \mathrm{et} \mathrm{al} \mathrm{.} \mathrm{Adipocyte-derived} \mathrm{exosomal} \mathrm{MiR-27a} \mathrm{induces} \mathrm{insulin}$ resistance in skeletal muscle through repression of PPAR $\gamma$. Theranostics 2018; 8: 2171.

86 Castaño C, Kalko S, Novials A, Párrizas M. Obesity-associated exosomal miRNAs modulate glucose and lipid metabolism in mice. Proceedings of the National Academy of Sciences 2018; 115: 12158-63.

87 Bai M, Chen H, Ding D, Song R, Lin J, Zhang Y, et al . MicroRNA-214 promotes chronic kidney disease by disrupting mitochondrial oxidative phosphorylation. Kidney international 2019; 95: 1389-404.

88 Rysz J, Gluba-Brzózka A, Franczyk B, Jabłonowski Z, Ciałkowska-Rysz A. Novel biomarkers in the diagnosis of chronic kidney disease and the prediction of its outcome. International journal of molecular sciences 2017; 18: 1702 .

89 Chau BN, Xin C, Hartner J, Ren S, Castano AP, Linn G, et al . MicroRNA-21 promotes fibrosis of the kidney by silencing metabolic pathways. Sci Transl Med 2012; 4: 121ra18-ra18.

90 Gomez IG, MacKenna DA, Johnson BG, Kaimal V, Roach AM, Ren S, et al . Anti-microRNA-21 oligonucleotides prevent Alport nephropathy progression by stimulating metabolic pathways. J Clin Invest 2015; 125: 141-56.

91 Fierro-Fernández M, Miguel V, Márquez-Exposito L, Nuevo-Tapioles C, Herrero JI, Blanco-Ruiz E, et al . MiR-9-5p protects from kidney fibrosis by metabolic reprogramming. The FASEB Journal 2020; 34: 410-31.

92 Cao M, Bai L, Wang D, Zhai Q, Li Y, Hai J, et al . miRNA-33 expression and its mechanism in patients and model rats with type 2 diabetic nephropathy. Int J Clin Exp Med 2018; 11: 1661-8.

93 Price NL, Miguel V, Ding W, Singh AK, Malik S, Rotllan N, et al . Genetic deficiency or pharmacological inhibition of miR-33 protects from kidney fibrosis. JCI insight 2019; 4.

94 Wang P, Luo M-L, Song E, Zhou Z, Ma T, Wang J, et al . Long noncoding RNA lnc-TSI inhibits renal fibrogenesis by negatively regulating the TGF- $\beta /$ Smad3 pathway. Sci Transl Med 2018; 10: eaat2039.

95 Wang M, Wang S, Yao D, Yan Q, Lu W. A novel long non-coding RNA CYP4B1-PS1-001 regulates proliferation and fibrosis in diabetic nephropathy. Molecular and cellular endocrinology 2016; 426: 136-45.

96 Wang J, Pan J, Li H, Long J, Fang F, Chen J, et al . IncRNA ZEB1-AS1 was suppressed by p53 for renal fibrosis in diabetic nephropathy. Molecular Therapy-Nucleic Acids 2018; 12: 741-50.

97 Long J, Badal SS, Ye Z, Wang Y, Ayanga BA, Galvan DL, et al . Long noncoding RNA Tug1 regulates mitochondrial bioenergetics in diabetic nephropathy. J Clin Invest 2016; 126: 4205-18.

98 Nastase MV, Zeng-Brouwers J, Wygrecka M, Schaefer L. Targeting renal fibrosis: Mechanisms and drug delivery systems. Advanced Drug Delivery Reviews 2018; 129: 295-307.

99 Huang K-C, Su Y-C, Sun M-F, Huang S-T. Chinese Herbal Medicine Improves the Long-Term Survival Rate of Patients With Chronic Kidney Disease in Taiwan: A Nationwide Retrospective Population-Based Cohort Study. Frontiers in Pharmacology 2018; 9. 
100 Zhang Y, Xu Y, Qi Y, Xu L, Song S, Yin L, et al . Protective effects of dioscin against doxorubicininduced nephrotoxicity via adjusting FXR-mediated oxidative stress and inflammation. Toxicology 2017; 378: $53-64$.

101 Cai S, Chen J, Li Y. Dioscin protects against diabetic nephropathy by inhibiting renal inflammation through TLR4/NF- $\varkappa$ B pathway in mice. Immunobiology 2020: 151941.

102 Zhao X, Yin L, Fang L, Xu L, Sun P, Xu M, et al . Protective effects of dioscin against systemic inflammatory response syndromevia adjusting TLR2/MyD88/NF- $x$ b signal pathway. International Immunopharmacology 2018; 65: 458-69.

103 Qiao Y, Xu L, Tao X, Yin L, Qi Y, Xu Y, et al . Protective effects of dioscin against fructose-induced renal damage via adjusting Sirt3-mediated oxidative stress, fibrosis, lipid metabolism and inflammation. Toxicology Letters 2018; 284: 37-45.

104 Wang M, Yang L, Yang J, Wang C. Shen Shuai IIRecipe attenuates renal injury and fibrosis in chronic kidney disease by regulating NLRP3 inflammasome and Sirt1/Smad3 deacetylation pathway. BMC complementary and alternative medicine 2019; 19: 1-9.

105 Chen D-Q, Hu H-H, Wang Y-N, Feng Y-L, Cao G, Zhao Y-Y. Natural products for the prevention and treatment of kidney disease. Phytomedicine 2018; 50: 50-60.

106 Layal K, Perdhana I, Louisa M, Estuningtyas A, Soetikno V. The effects of quercetin on oxidative stress and fibrosis markers in chronic kidney disease rat model. Medical Journal of Indonesia 2017; 26: 169.

107 Cao Y, Hu J, Sui J, Jiang L, Cong Y, Ren G. Quercetin is able to alleviate TGF- $\beta$-induced fibrosis in renal tubular epithelial cells by suppressing miR-21. Experimental and therapeutic medicine 2018; 16: $2442-8$.

108 Liu X, Sun N, Mo N, Lu S, Song E, Ren C, et al . Quercetin inhibits kidney fibrosis and the epithelial to mesenchymal transition of the renal tubular system involving suppression of the Sonic Hedgehog signaling pathway. Food \& function 2019; 10: 3782-97.

109 Jiang X, Yu J, Wang X, Ge J, Li N. Quercetin improves lipid metabolism via SCAP-SREBP2-LDLr signaling pathway in early stage diabetic nephropathy. Diabetes, metabolic syndrome and obesity: targets and therapy $2019 ; 12: 827$.

110 Tasatargil A, Tanriover G, Barutcigil A, Turkmen E. Protective effect of resveratrol on methylglyoxalinduced endothelial dysfunction in aged rats. Aging clinical and experimental research 2019; 31: 331-8.

$111 \mathrm{Li} \mathrm{H}$, Xia N, Hasselwander S, Daiber A. Resveratrol and vascular function. International journal of molecular sciences 2019; 20: 2155.

112 Cosín-Tomàs M, Senserrich J, Arumí-Planas M, Alquézar C, Pallàs M, Martín-Requero Á, et al . Role of Resveratrol and Selenium on Oxidative Stress and Expression of Antioxidant and Anti-Aging Genes in Immortalized Lymphocytes from Alzheimer's Disease Patients. Nutrients 2019; 11: 1764.

113 Lagouge M, Argmann C, Gerhart-Hines Z, Meziane H, Lerin C, Daussin F, et al . Resveratrol Improves Mitochondrial Function and Protects against Metabolic Disease by Activating SIRT1 and PGC- $1 \alpha$. Cell 2006; 127: 1109-22.

114 Kim MY, Lim JH, Youn HH, Hong YA, Yang KS, Park HS, et al . Resveratrol prevents renal lipotoxicity and inhibits mesangial cell glucotoxicity in a manner dependent on the AMPK-SIRT1-PGC1 $\alpha$ axis in db/db mice. Diabetologia 2013; 56: 204-17.

115 Voloshyna I, Hai O, Littlefield MJ, Carsons S, Reiss AB. Resveratrol mediates anti-atherogenic effects on cholesterol flux in human macrophages and endothelium via PPAR $\gamma$ and adenosine. European Journal of Pharmacology 2013; 698: 299-309. 
116 Zhou Y, Lin S, Zhang L, Li Y. Resveratrol prevents renal lipotoxicity in high-fat diet-treated mouse model through regulating PPAR- $\alpha$ pathway. Molecular and cellular biochemistry 2016; 411: 143-50.

117 Liu S, Zhao M, Zhou Y, Wang C, Yuan Y, Li L, et al . Resveratrol exerts dose-dependent anti-fibrotic or pro-fibrotic effects in kidneys: A potential risk to individuals with impaired kidney function. Phytomedicine 2019; 57: 223-35.

118 Kheirollahi V, Wasnick RM, Biasin V, Vazquez-Armendariz AI, Chu X, Moiseenko A, et al . Metformin induces lipogenic differentiation in myofibroblasts to reverse lung fibrosis. Nature communications 2019; 10: $1-16$.

119 Yi H, Huang C, Shi Y, Cao Q, Zhao Y, Zhang L, et al . Metformin attenuates folic-acid induced renal fibrosis in mice. Journal of cellular physiology 2018; 233: 7045-54.

120 Ding H, Jiang L, Xu J, Bai F, Zhou Y, Yuan Q, et al . Inhibiting aerobic glycolysis suppresses renal interstitial fibroblast activation and renal fibrosis. Am J Physiol Renal Physiol 2017; 313: F561-F75.

121 Li M, Jia F, Zhou H, Di J, Yang M. Elevated aerobic glycolysis in renal tubular epithelial cells influences the proliferation and differentiation of podocytes and promotes renal interstitial fibrosis. Eur Rev Med Pharmacol Sci 2018; 22: 5082.

122 Wei Q, Su J, Dong G, Zhang M, Huo Y, Dong Z. Glycolysis inhibitors suppress renal interstitial fibrosis via divergent effects on fibroblasts and tubular cells. American Journal of Physiology-Renal Physiology 2019; 316: F1162-F72. 


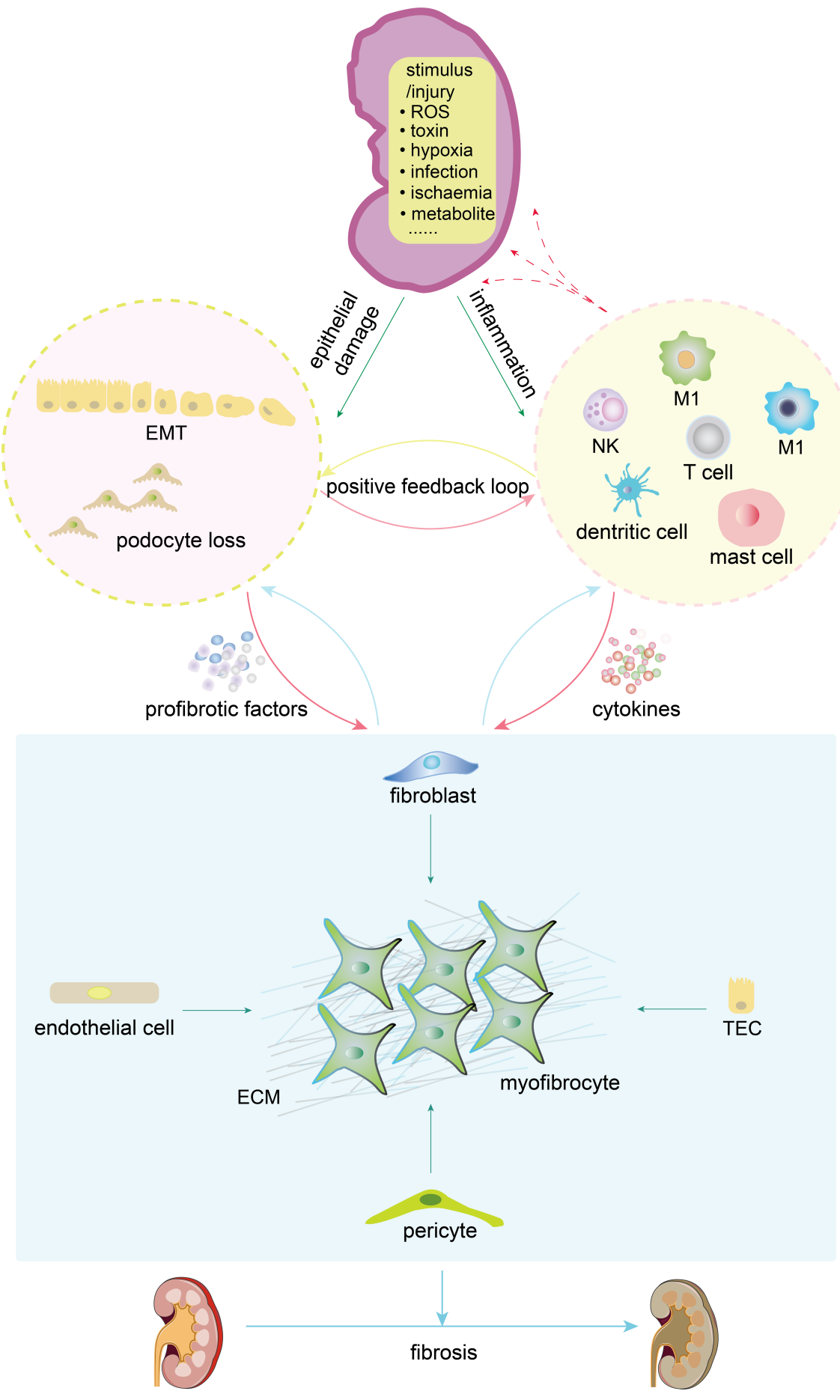




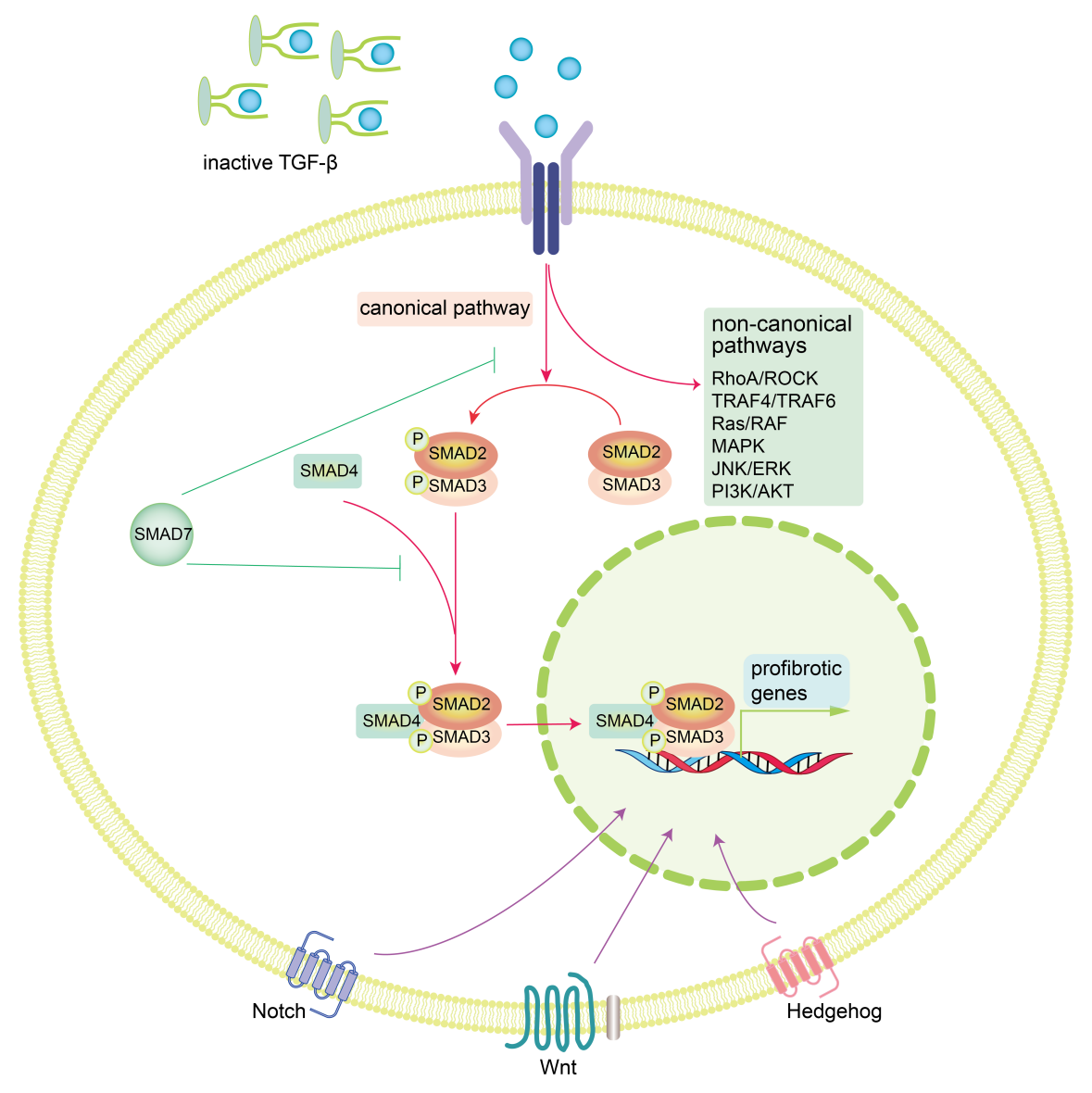



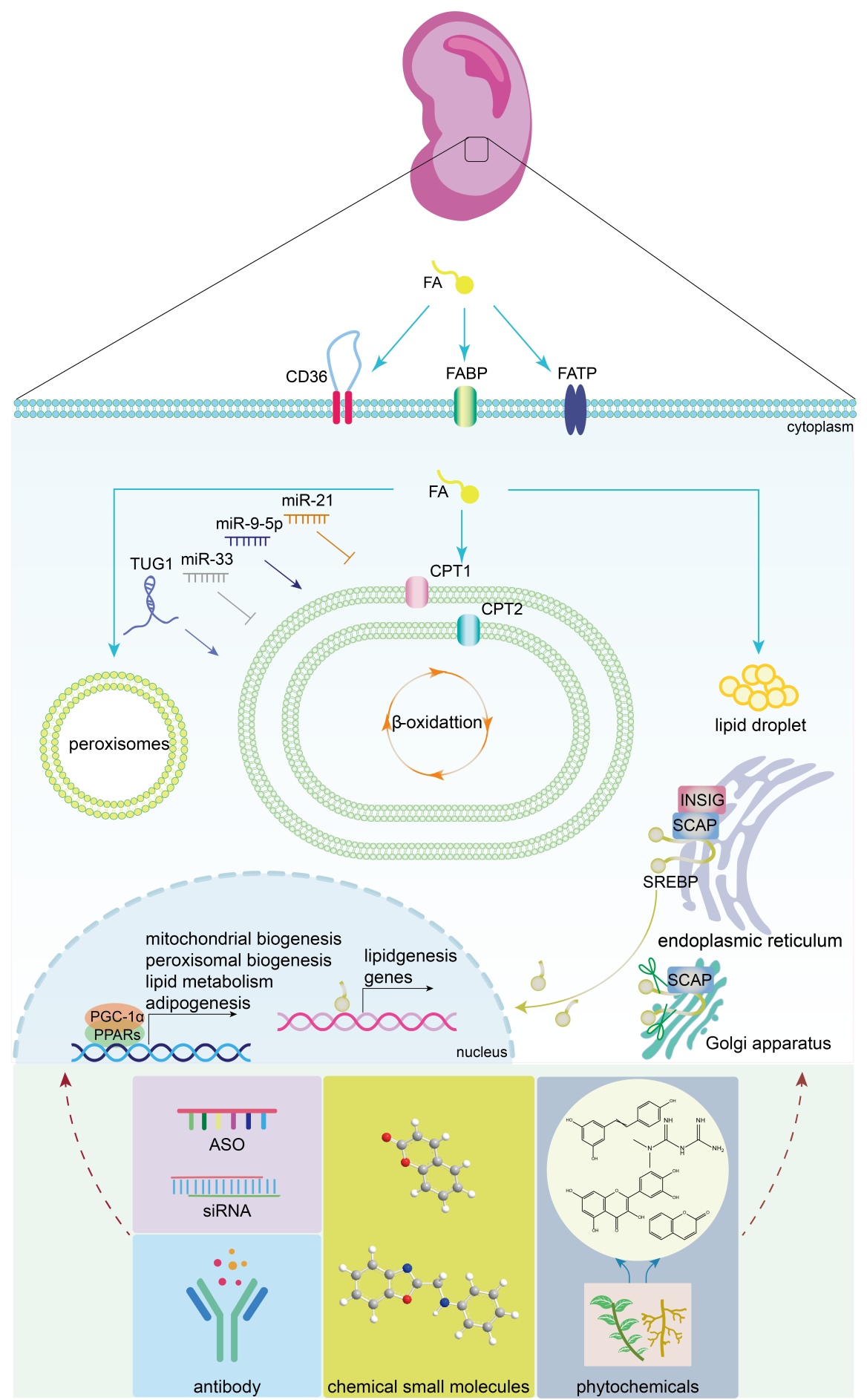

\section{Hosted file}

Figure Legend.docx available at https://authorea.com/users/327213/articles/454845-freshinsight-polish-the-druggability-of-lipid-metabolism-for-anti-renal-fibrosis

\section{Hosted file}


Table 1.docx available at https://authorea.com/users/327213/articles/454845-fresh-insightpolish-the-druggability-of-lipid-metabolism-for-anti-renal-fibrosis

\section{Hosted file}

Table 2.docx available at https://authorea.com/users/327213/articles/454845-fresh-insightpolish-the-druggability-of-lipid-metabolism-for-anti-renal-fibrosis

\section{Hosted file}

Table Legend.docx available at https://authorea.com/users/327213/articles/454845-freshinsight-polish-the-druggability-of-lipid-metabolism-for-anti-renal-fibrosis 